\title{
SARCOCAPNOS BAETICA (BOISS. \& REUTER) NYMAN SUBSP. ARDALII G. LÓPEZ VÉLEZ, SUBESPECIE NUEVA PARA LA PENÍNSULA IBÉRICA
}

\author{
Gemma LÓPEZ VÉLEZ
}

RESUMEN. Se describe una nueva subespecie para la flora ibérica: Sarcocapnos baetica (Boiss. \& Reuter) Nyman, subsp. ardalii (subsp. nov.) (Papaveraceae), que ha sido estudiada desde el punto de vista morfológico, ecológico y corológico. Se aportan datos comparativos que permiten establecer diferencias entre el nuevo taxon y Sarcocapnos baetica subsp. baetica, en el que hasta ahora había sido incluido y S.baetica subsp. integrifolia (Boiss.) Nyman, con la que presenta algunas semejanzas en sus frutos, a menudo monospermos.

Palabras clave. Papaveraceae, Sarcocapnos, España.

SUMMARY. A new subspecies for the Iberic Flora is described: Sarcocapnos baetica (Boiss. \& Reuter) Nyman subsp. ardalii (subsp. nov.) (Papaveraceae) which is studied under morphological, ecological and chorological points of view. Comparative data are given. They allow to establish differences between the new taxa and Sarcocapnos baetica subsp. baetica in which it has been included until now, and S. baetica subsp. integrifolia (Boiss.) Nyman, compared to the latter it shows some similarities in the fruit, usually beeing monospermous.

Key words. Papaveraceae, Sarcocapnos, Spain.

En el curso de los estudios que estamos realizando sobre la flora de la comarca de Yeste (Albacete), hemos detectado una planta relacionada con Sarcocapnos baetica (Boiss. \& Reuter) Nyman, que se presenta densamente pelosa de un color verde tenue, y con sus hojas mayoritariamente trifoliadas, caracteres estos que la diferencian de la subsp. baetica típica. Tambien nos llamó la atención que siendo espelúncicola, no coexiste con táxones de su género. Durante las herborizaciones realizadas con objeto de hacer un estudio de su distribución, localizamos otras dos zonas, incluidas en la comarca de Yetas de Abajo (Albacete).

De la revisión del material depositado en el Herbario de Madrid, pudimos comprobar que existe un pliego con ejemplares de las mismas caracteristicas de los nuestros, recogido en la Sierra de Guillimona (Granada) por Leal (1978) en pliego de Fernández Casas (MA 231.525) incluida en la misma especie. Dicho pliego presenta 

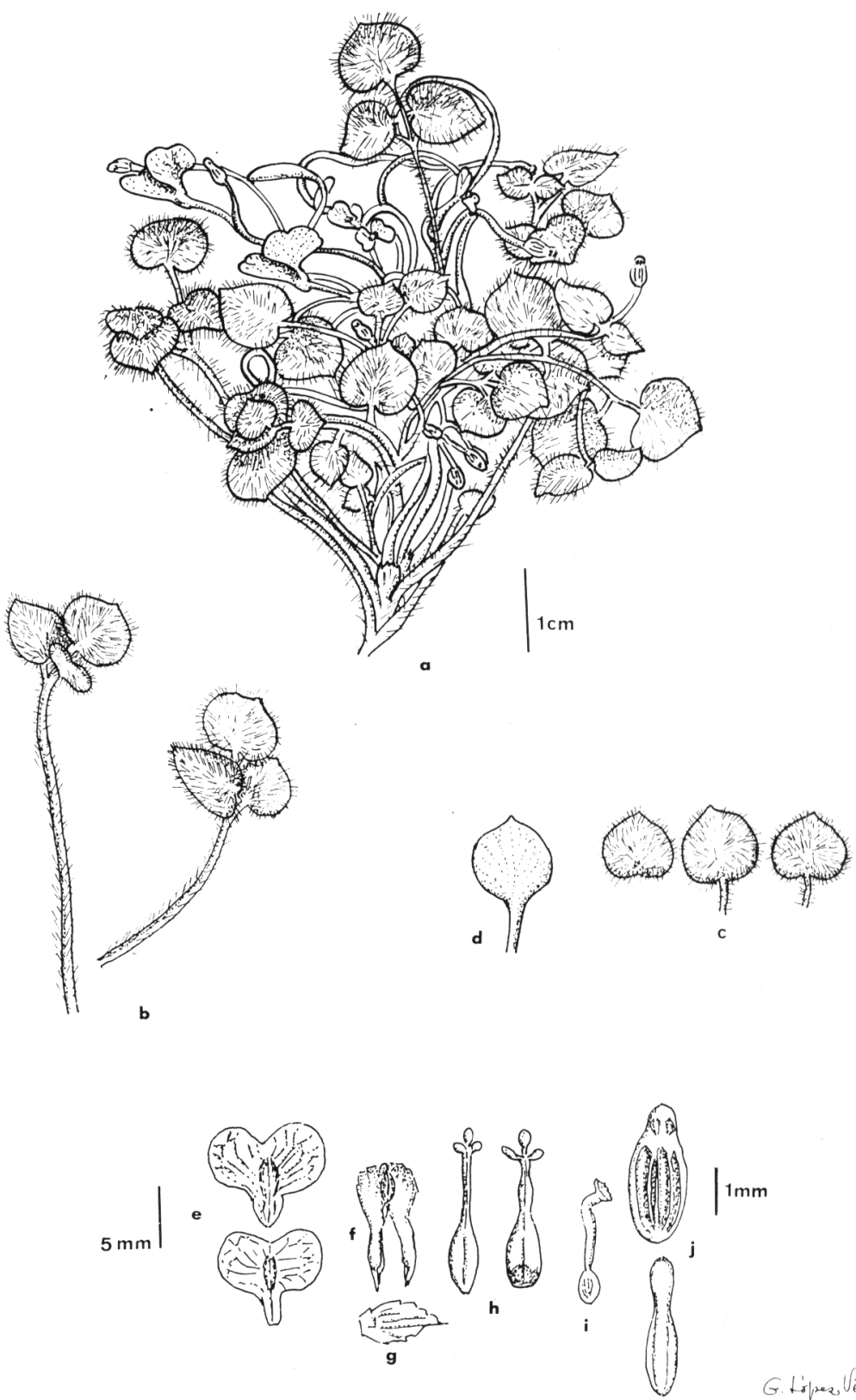

G.tiper, vele

Fig. 1. Sarcocapnos baetica subsp. ardalii. Cerro de Rala (Yeste). Albacete (MUB 6665): a) habito, b) hoja trifoliada velloso-hirsuta; c) foliolos de base cordada; d) partes de la flor; e) pétalos superior e inferior; f) pétalos internos; h) estambres; i) gineceo; g) sépalo; j) fruto en posición frontal y lateral; k) foliolo comparativo de $S$. baetica subsp. baetica. 
una anotación de Magnus Lidén en etiqueta de 1983 en la que escribe: «Unique population !densely hairy!».

Como consecuencia del estudio de los mismos pudimos comprobar que se trata de táxones diferentes, debiendo ser excluida de la subsp. baetica y ser descritos en otra categoría subespecífica.

Sarcocapnos baetica (Boiss. \& Reuter) Nyman subsp. ardalii subsp. nov.

Describitur ut Sarcocapnos baetica (Boiss. \& Reuter) Nyman, alia subespecies. Differt ab exemplari cum sit minor, setis pilosa in rachis, petiolis, et foliolis, colore non nimis viridi; folia trifoliata, floris maiori magnitudine, cum petalo superiore et inferiore marginato et prolato. Fructus monospermi sine funiculo et sustantia oleosa madefacti. Minore numero cromosomico.

Holotypus: Prope Yeste, Albacete, loco dicto Cerro de Rala, in rupibus calcareis abruptis et superverticalibus, 30SWH6352 ad 800 m, 3-XI-1982, ub runt G.López Vélez. MUB 6665.

Isotypus: Enviados a los herbarios MA, BC.

El nombre «Ardalii» hace referencia a la sierra de Ardal (Yeste), localidad donde fué recogida.

Se trata de un caméfito cespitoso (fig. I,a) perenne, que según los individuos alcanza entre los 5 y $13 \mathrm{~cm}$ de longitud, de tallos gruesos semileñosos con cepas viejas persistentes. Es de un color verde tenue, densamente pelosa. Las hojas son mayoritariamente trifoliadas (fig. 1,b,c) con foliolos velloso-hirsutos, de ovado redondeado a cordados en su base, y generalmente con mucrón pronunciado. El foliolo impar por lo general más grande $(7 \times 5 \mathrm{~mm})$ que los dos opuestos $(5 \times 4 \mathrm{~mm})$. Peciólulos entre $0.2,0.5$ y $5 \mathrm{~cm}$.

El indumento está constituido por tricomas blancos de hasta $3 \mathrm{~mm}$ de longitud, sin articular y de porte cilindrico en fresco (fig. 2,b). Se encuentran dispuestos densamente sobre las hojas y tallos, especialmente en los brotes jóvenes, aunque tambien se conservan en los secos. Los tallos floríferos y fructíferos no son pubescentes.

Las flores son largamente pedunculadas, zigomorfas, blancas, de hasta $8 \mathrm{~mm}$ de largo por $5 \mathrm{~mm}$ de ancho, presentan el pétalo superior con escotadura pronunciada y prolongado, así como el inferior, aunque menos. Pétalos internos más cortos, auriculados y con mancha amarilla en sus extremos. Sin espolón. Sépalos petaloideos romboidales y serrados. Estambres dos, cada uno con tres anteras, y el superior con nectario basal que encaja en el ensanchamiento que presenta la parte inferior del pétalo superior, confundiendose con un rudimento de espolón. Estigma sin cresta plana, con estilo grueso y fuerte (fig. 1,i). Frutos geocarpicos de hasta $3 \mathrm{~mm}$ de largo por $1 \mathrm{~mm}$ de ancho. Costillas laterales más anchas que las centrales, pico abombado lateralmente, obtuso y engrosado en su ápice, con dos poros «germinativos» grandes. Tendencia monosperma o con rudimentos de otra semilla sin desarrollar. 
Semillas de forma ovada (fig. 3, foto a) de hasta $2 \mathrm{~mm}$ de largo por $1 \mathrm{~mm}$ de ancho, impregnadas siempre de una sustancia oleosa. Cuando se desarrollan dos, presentan su base plana como en otras especies de Sarcocapnos. Sin funículo prominente, hilo invaginado hacia dentro, texta de color marrón, reticulada, con retículos rectangulares y endopleura espiculada (fig. 3, fotos b,c).

Número cromosómico $2 \mathrm{n}=24$ (recuento realizado a partir de una mitosis somática de tejidos florales por M.Bernal, 1984, (Barcelona).

Florece desde mediados de Mayo a mediados de Junio.

Ecología: Planta rupícola, habita en fisuras de rocas y grietas de paredes superverticales calizo-dolomíticas, a veces de facil disgregación. Vive en altitudes entre los 800 y $1700 \mathrm{~m}$. con ombroclima seco y con orientación preferente a N o NE. Se desarrolla en comunidades de Saxifragion camposii Cuatrecasas, 1929, en los pisos Supramediterráneo y Oromediterráneo (Tabla 1). Más raramente baja al horizonte superior del piso Mesomediterráneo, en comunidades de Teucrion buxifolii Rivas Goday 1955, donde convive con Hypericum ericoides, Chiladenus glutinosus, Fumana ericoides subsp. montana, Sanguisorba ancistroides, Polygala rupestris, entre otras.

\section{TABLA 1}

Comunidad de SARCOCAPNOS BAETICA subsp. ARDALII

$\mathrm{n}^{\mathrm{o}}$ Orden
Altitud m
Inclinación
Orientación
Area $\mathrm{m}^{2}$
$\mathrm{~N}^{\mathrm{o}}$ de especies

Caracteristicas de alianza y unidades superiores:

Sarcocapnos baetica subsp. ardalii

Saxifraga camposii

Teucrium rotundifolium

Arenaria grandiflora

Sedum acre

Draba hispanica

Erodium cheilantifolium

Silene saxifraga

Compañeras:

Thymus orospedanus

Satureja obovata subsp. obovata

$\begin{array}{cc}1 & 2 \\ 1300 & 1650 \\ 90^{\circ} & 90^{\circ} \\ \mathrm{NW} & \mathrm{NE} \\ 9 & 10 \\ 6 & 8\end{array}$

$\begin{array}{cc}2.2 & 3.3 \\ 1.1 & 2.2 \\ 1.1 & 1.1 \\ + & 1.1 \\ . & 1.1 \\ . & 1.1 \\ . & 1.1 \\ . & +\end{array}$

$+$

$+$

Localidades: 1.- Puerto del Pino, 30SWH3914, Santiago de la Espada (J), calizas dolomíticas; 2.- Los Voladores, 30SWH4643, Yeste (Ab), Paredón calizo. 


\begin{tabular}{|c|c|c|}
\hline & S. baetica & S. ardalii \\
\hline Hábito & $\begin{array}{l}\text { Casmófito denso, glabro, } \\
\text { pulviniforme o péndulo de } \\
\text { color verde fuerte, entre } 5 \mathrm{y} \\
10 \mathrm{~cm} \text {.(variable). }\end{array}$ & $\begin{array}{l}\text { Casmófitodensamente pubes- } \\
\text { cente pulviniforme y de un } \\
\text { verde apagado, entre } 5 \text { y } 13 \\
\mathrm{~cm} \text {. (variable). }\end{array}$ \\
\hline Hojas y peciolos & $\begin{array}{l}\text { Glaucos, con 1-2-6-7 } \\
\text { segmentos, ovado redondea- } \\
\text { dos mucronados Peciolos } \\
\text { largos. }\end{array}$ & $\begin{array}{l}\text { Densamente pelosos, velloso- } \\
\text { hirsutos, trifoliados, foliolos } \\
\text { cordados en su base, el impar } \\
\text { de hasta } 7 \text { x } 5 \text { mm } \\
\text { mucronados. Peciolos largos. }\end{array}$ \\
\hline Indumento & No tiene. & $\begin{array}{l}\text { Pelos blanquecinos, cilíndri- } \\
\text { cos no articulados y de hasta } 3 \\
\text { mm de longitud, no presentes } \\
\text { en los tallos floríferos ni } \\
\text { fructíferos. }\end{array}$ \\
\hline
\end{tabular}

Flores

Fruto

Semilla

№ cromosómico $\quad 2 \mathrm{n}=32$
Zigomorfas, blancas, de 5 a 6 $\mathrm{mm}$. Pétalos por lo general más redondeados. Con espolón rudimentario.

Oblongo,, comprimido, con nervios subiguales, por 10 general dispermo, de 3-4 x 2 $\mathrm{mm}$.

Ordinariamente dos granos, redondeados y planos en la base que contactan entre sí ambas semillas. Funículo patente membranoso. Tegumento ligeramente punteado. Granos de 1 x $1 \mathrm{~mm}$.
Zigomorfas, blancas, de hasta 8 x $5 \mathrm{~mm}$. Pétalos escotados y dilatados. El superior con base ensanchada, conteniendo la glándula estaminal del estambre superior. Sin espolón.

Oval comprimido con nervios subiguales, mayoritariamente monospermo de hasta $3 \times 1$ $\mathrm{mm}$.

Ordinariamente 1 solo grano, obado, oblongo, sin funículo hilo con reticulaciones, lineares, tegumento marrón brillante, envuelta en un líquido aceitoso y con pequeñas reticulaciones rombo rectangulares. Granos de $2 \times 1 \mathrm{~mm}$, a veces aparecen rudimentos de otra sin desarrollar.
S. integrifolia

Casmófito denso, glabro, pulviniforme de color verde fuerte, cepas viejas gruesas, tamaño variable.

Glaucos, hojas enteras, cuneadas en la base y muy carnosas-mucronadas Peciolos gruesos.

No tiene.

Zigomorfas, blanquecinas mucho más pequeñas. Pétalos sin escotadura y muy prolongados sin espolón.

Oval-oblongo y con tendencia monosperma, $3 \mathrm{~mm}$.

Frecuentemente 1 solo grano, redondeado, con funículo patente, con la texta con reticulación liniar. Granos de $1,5 \mathrm{~mm}$

Desconocido.

Tabla 2. Caracteres diferenciales entre S. baetica, S. ardalii y S. integrifolia. 

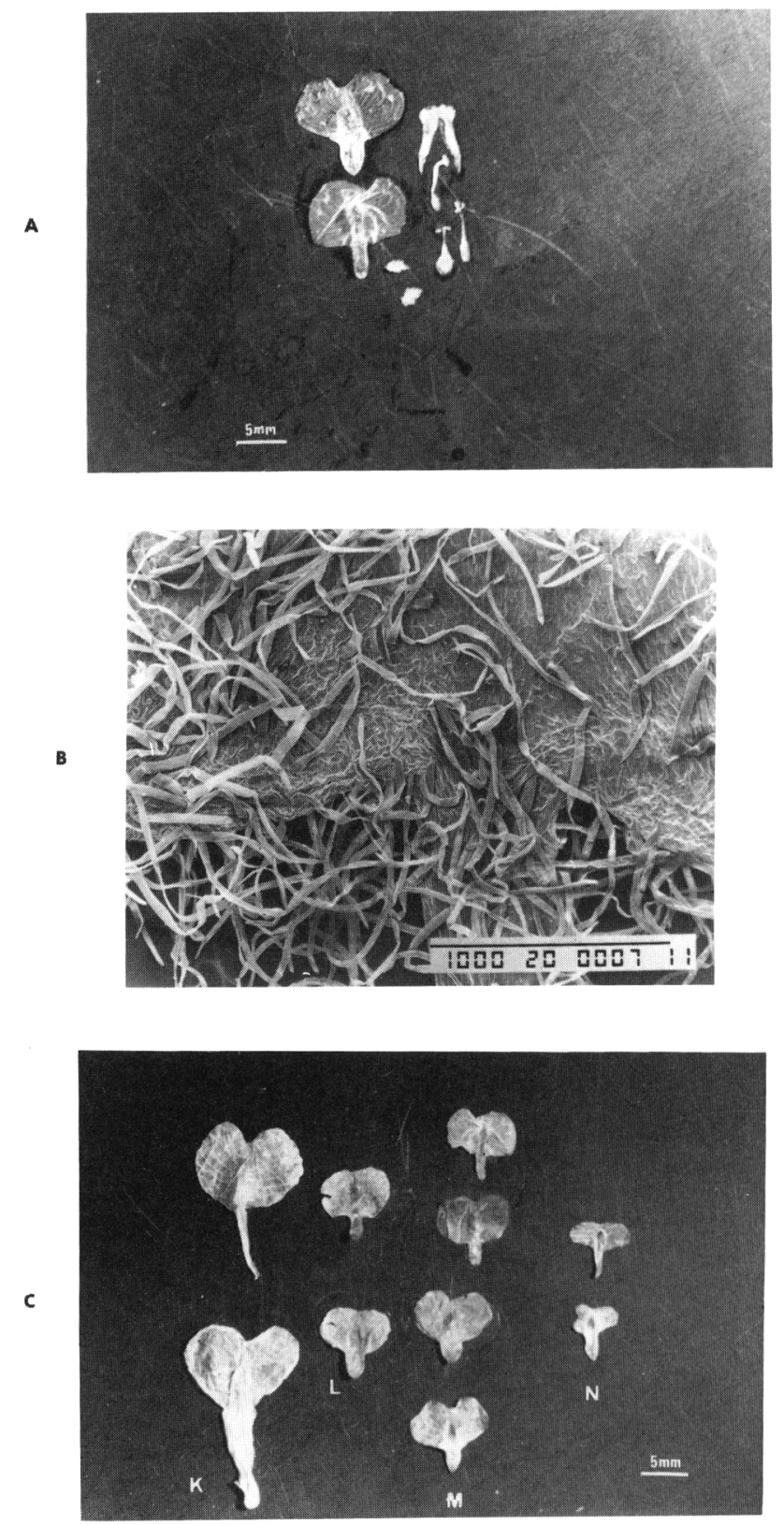

Fig. 2. A) flor diseccionada de S. baetica subsp. ardalii; B) microfotografía electrónica de la base de la hoja y peciolo, con indumento de pelos cilíndricos, no articulados. Escala 1000 micras; C) Tamaños y formas de pétalos de: K) Sarcocapnos crasifolia; L) S. baetica subsp. baetica; M) S. baetica subsp. ardalii; N) S. baetica subsp. integrifolia. 

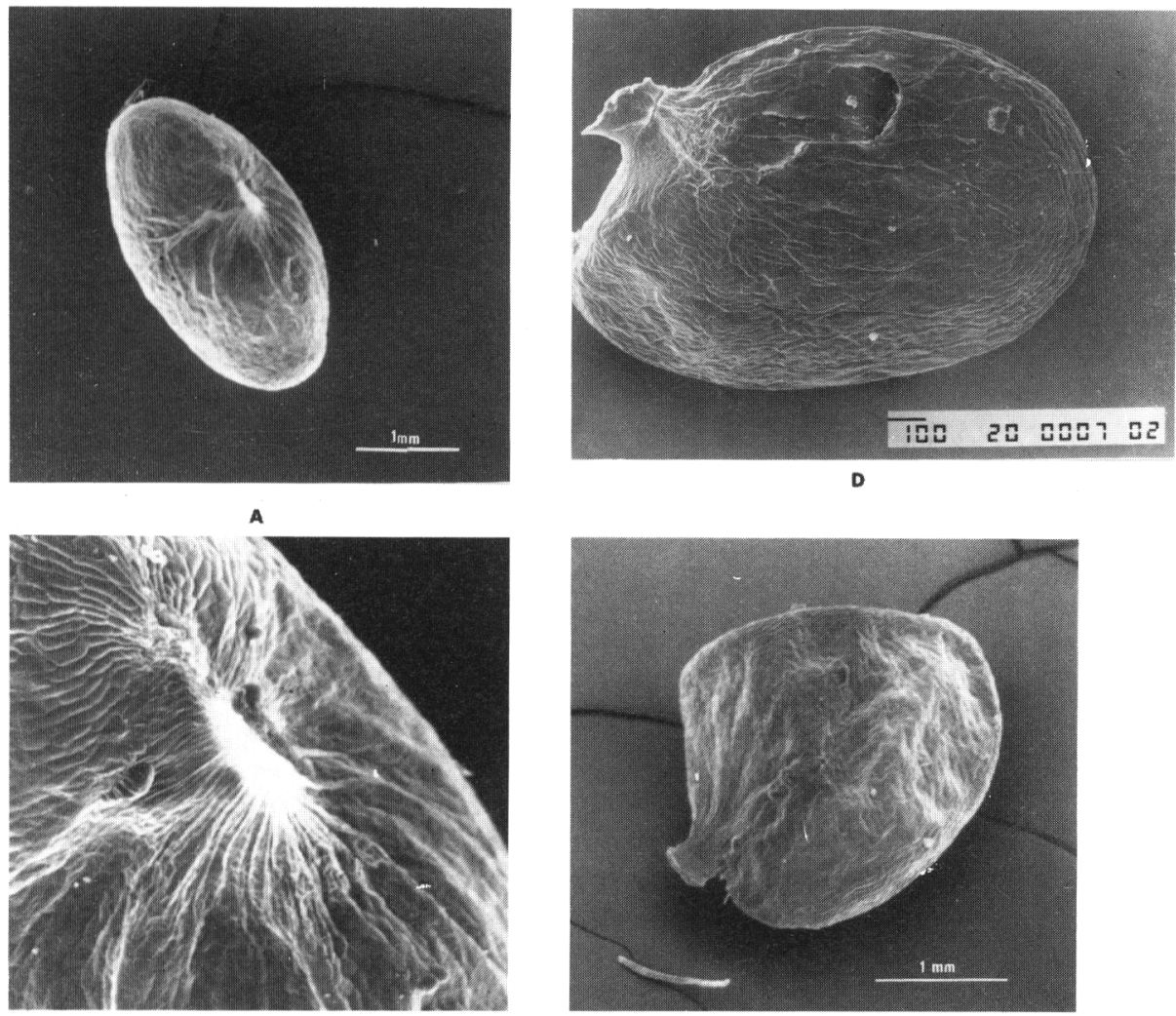

B

E

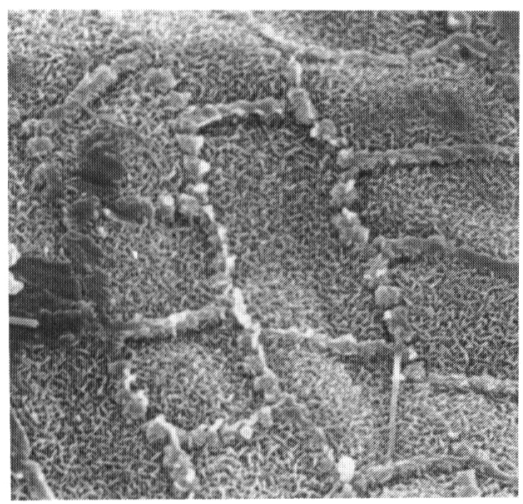

c

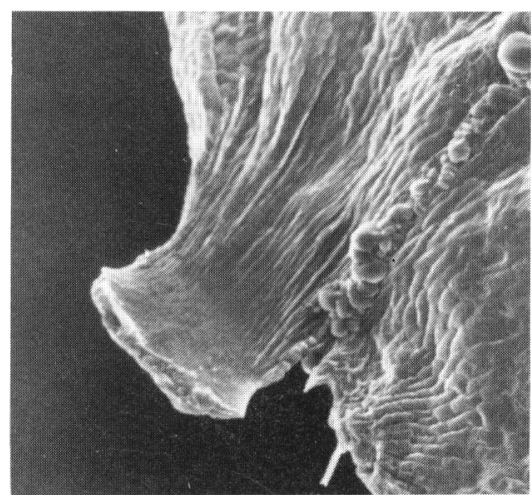

G

Fig. 3. Sarcocapnos baetica subsp. ardalii: a) forma esterna de la semilla; b) detalle del hilo (X 200); c) detalle de la texta y endopleura con prominencias espiculadas (X 1000); d) semilla de S. baetica subsp. integrifolia. Escala 100 micras. Sarcocapnos baetica subsp. baetica; e) semilla; g) detalle del funiculo (X 200). 
Corología: Detallamos a continuación el material estudiado, lo que nos permite establecer aproximadamente su distribución: (fig. 4, mapa del SE en P. Ibérica).

ALBACETE: Cerro de Rala (Yeste), WH6352, 800 m, fisura de rocas calizodolomíticas. 8-5-1983; MUB 6679, G. López Vélez. Ibidem, 6-6-1983, MUB 30453, G. López Vélez. Cerro de La Molata del Imperio (Yeste), WH4643, 1400 m, paredón vertical extraplomado, 5-6-1981, MUB 30449, G. López Vélez. Los Voladores (Calar de la Sima) Yetas de Abajo, WH4642, 1700 m, paredones extraplomados, calizos, 56-1982, MUB 30449, G.López Vélez. JAEN: Rio Zumeta (Proximidades de Santiago de la Espada), WH4825, 900 m, roquedos calizos, 19-5-1983; MUB 30448, G. López Vélez. GRANADA: Sierra de Guillimona (Barranco de los Pastores), WH3843, 1500 $\mathrm{m}$, in rupibus calcareis verticalibus, 21-5-1978; Leal, 294, MA 231525, Fernandez Casas.

Se trata de un endemismo ibérico de las Sierras subbéticas orientales, cuya distribución estudiada hasta el momento se limita a algunas áreas de las provincias de Albacete, Jaén y Granada incluidas en la provincia corológica Bética.

Consideramos probable su presencia en la Sierra de Harana y en el Puerto del Pinar, ambas localidades en la provincia de Granada (Gandoger, 1917), localidades que tenemos por confirmar en proximos desplazamientos.

Observaciones: Se ha realizado un estudio comparativo, detallado, entre este nuevo taxon, y otros de Sarcocapnos (S.baetica subsp. baetica y S.baetica subsp. integrifolia) presentes en las sierras béticas y con las que mantiene mayor afinidad.

Aunque S.eneaphylla y S.crassifolia subsp. speciosa, tambien tienen su menor o mayor presencia en estas áreas, las diferencias interespecíficas son tan notables que no ofrece duda alguna sobre su separación.

Las diferencias entre las tres subespecies se presentan de modo resumido en la tabla 2, con el fín de facilitar su discriminación.

Existe variabilidad en el número y medida de las flores, forma, y dimensiones de los foliolos, lo cual también es característico de todos los táxones de Sarcocapnos. Por ello se han elegido aquellas formas foliares y florales que son más constantes. Los caracteres establecidos como diferenciales entre las tres subespecies de Sarcocapnos, proceden del estudio sobre pliegos facilitados por los herbarios MA y MUB y que a continuación reseñamos:

Sarcocapnos baetica (Boiss. \& Reuter) Nyman subsp. baetica : Sierra del Ardal, WH5546, alt 1300 m, paredón extraplomado; 6-4-1980, G. López Vélez(MUB 6591). Carretera del Pardal a Molinicos, WH6548, alt 960 m, paredón vertical; 25-51981, G. López Vélez (MUB 30454). Fuente de la Majada del Soto (Cotillas), WH4453, alt 1000 m, 6-7-1980, G. López Vélez (MUB 6590).

Sarcocapnos baetica subsp. ardalii : Leal. Loc. cit. (MA 231525), las muestras de este pliego al estar recogidas con fecha 21-5-1978, no presentan frutos formados y sólo se pudo analizar la flor y la hoja. MUB 6679. MUB 30449. MUB 30448. 


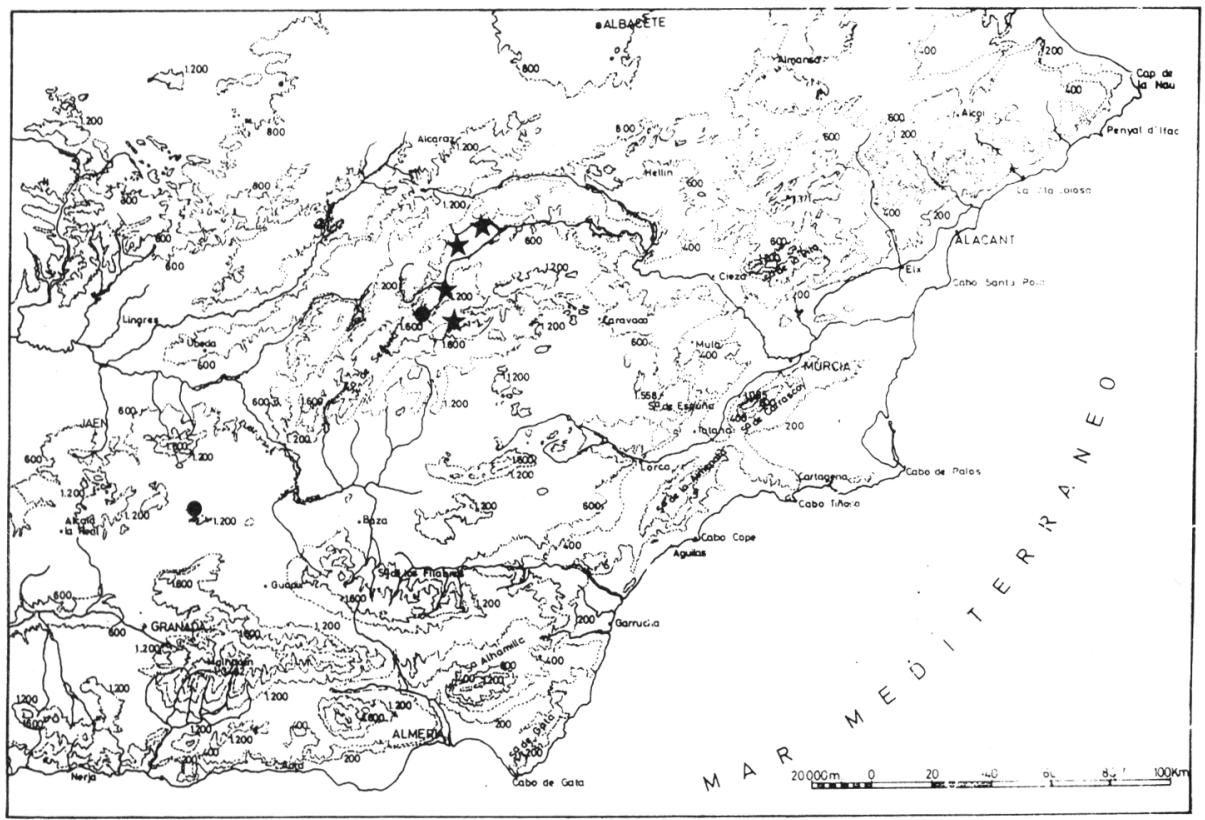

A

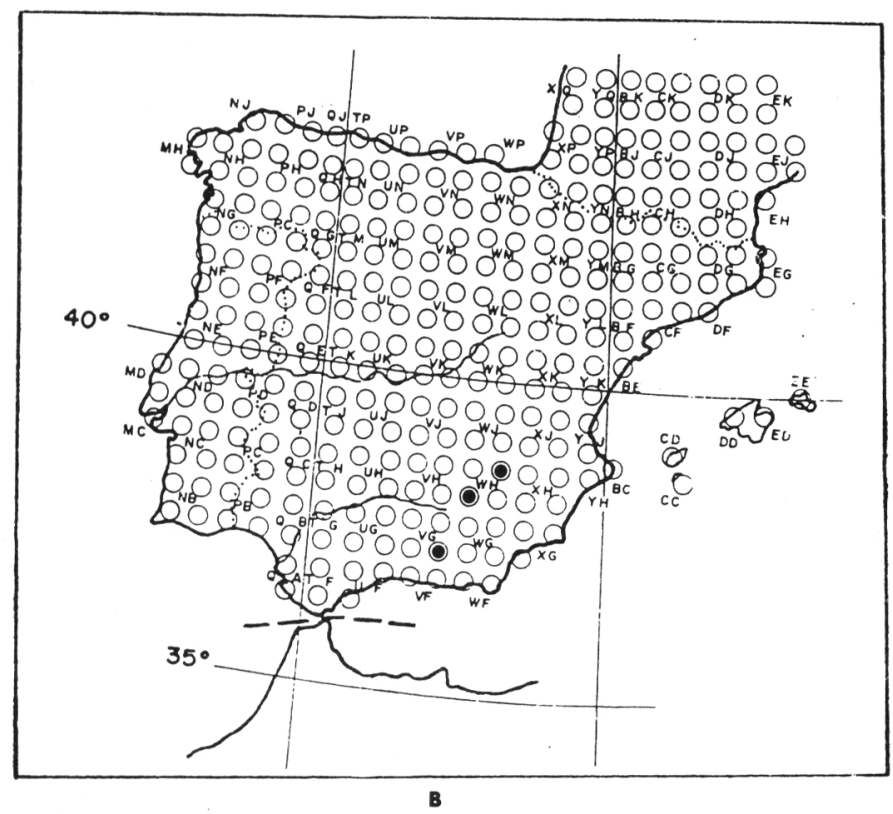

Fig. 4. A) Mapa del SE, en la Península Ibérica: Distribución de las localidades para las provincias de Albacete y Jaen. Localidades citadas por Gandoger. B) Mapa de coordenadas UTM. 
Sarcocapnos baetica subsp. integrifolia: Herb. Hort. Reg. Matrit. Reverchon. Plantas de España 1908. Sarcocapnos baetica var. integrifolia Boiss. \& Reuter. Sierra del Cuarto (Granada), alt 1700 m, Junio (MA 42970). Sarcocapnos integrifolia (Boiss.) Cuatrecasas. Fernández Casas. Fisura de rocas calizas en el conjunto de la Cerradura, carretera Jaén-Granada (Jaén). 15-7-1971; J.Molero \& F.Casas (MA 423836). Herbarium Cuatrecasas. Flora Hispanica. Sarcocapnos integrifolia (Boiss.) Cuatrecasas. La Silleta. Declin.NW Sierra de la Cabra del $\mathrm{St}^{\circ}$ Cristo (Macizo de Mágina, Jaén). In Rupestri, alt 1200 m. calcareis 10-6-1925 (MA 42971).

Estas poblaciones de Sarcocapnos baetica subsp. ardalii, parece que fueron observadas y recolectadas por Reverchon y Gandoger en 1901, como consta en el exsiccato de este último: «Iter Hispanicum 1908. M.Gandoger» depositados en Arnas (Rhône), Francia. Entre sus pliegos, existe uno sin numerar que contiene un ejemplar y parte de otro sin tipificar, en un estado de conservación deficiente, pero en el que se pueden reconocer las características de los nuestros. Dichos ejemplares están acompañados de tres etiquetas sin adherir, en dos de las cuales se puede leer el nombre que Gandoger le dedica (S.trifoliata) y la localidad de su recolección (Granada, La Sagra ad Sierra del Pinar, Julio 1901; legit E. Reverchon, alt 5-5500).

Sobre la segunda etiqueta, que parece de campo, se lee el mismo nombre: S.trifoliata, Hisp. Granada, Gandoger y E. Reverchon. En ella aparece una corrección en letra deficiente y sobrecruzando el anterior nombre que dice: №6, Sarcocapnos enneaphylla, Sierra del Pinar, 1800 m, Julliet 1901. Esta última corrección pone en evidencia si dichos ejemplares fueron posteriormente interpretados como S.enneaphylla.

Por otro lado, consultada la descripción que Gandoger (1917) hace de la misma en su «Catalogue des plantes Récoltéés en Espagne et en Portugal...etc, escrito de su puño y letra, aparece en la nota n⿳⺈ 271 , correspondiente al género Sarcocapnos DC. una descripción en la que mezcla caracteres de ambos táxones, asignándoselos a S.trifoliata: "Tota crispula villosa atrovirens diffusa, folia herbacea trifoliata, foliolis undique villosis... ...flores albidi minores, calcar breve rectum... ...fructus trinervius, late stilatus. Maio.»

Esta fusión de caracteres: («...espolón corto recto...») propio de S.eneaphylla, con los de («...Toda llena de pelo encrespado, de un verde apagado, hoja herbácea trifoliada...») propios de esta nueva subespecie, nos hace pensar que no llegó a interpretar y describir correctamente este taxon.

La tercera etiqueta es de Magnus Lidén 1980, el cual la incluye como Sarcocapnos baetica. Este autor (Lidén, 1986) comenta «La población de Sierra Guillimona es, sin embargo, densamente pelosa», atribuyendo los ejemplares de estos pliegos (Gandoger y Fernández Casas, MA 231525) a la subespecie baetica.

En base a todo lo anterior hemos realizado la siguiente clave donde se pueden diferenciar las tres subespecies del complejo Sarcocapnos baetica: 
1. Hojas enteras, cuneadas en la base (c) subsp. integrifolia

1'. Hojas generalmente divididas 2

2. Hojas con 1-6 (8) segmentos cordados en la base. Flores de 5-6 mm. Frutos de 3-4 x c.2 $\mathrm{mm}$. Totalmente glabra (a) subsp. baetica

2'. Hojas con tres segmentos, planta densamente pelosa, en raquis, peciolos y foliolos, de un color verde apagado. Flores de hasta $8 \mathrm{~mm}$ con pétalos escotados, fruto de hasta $3 \times 1 \mathrm{~mm}$, con tendencia monosperma (b) subsp. ardalii

AGRADECIMIENTOS. A F.Alcaraz, por su orientación y ayuda prestadas en la realización del trabajo. A D. Rivera por la facilitación del Exsiccato y manuscrito de Gandoger. A Dr.Rigueiro, conservador del Herbario del Jardín Botánico de Madrid por el rápido préstamo de los pliegos solicitados.

\section{BIBLIOGRAFIA}

GANDOGER, M. -1917- Catalogue des plantes récoltées en Espagne et en Portugal pendant mes voyages de 1894 a 1912. Paris.

GREUTER, W., H.M. BURDET, \& G. LONG -1989-Med-Checklist. Vol. IV. Dicotyledones (Lauraceae-Rhamnaceae). Eds. Conserv. Jard. Bot. Genéve.

LIDEN, M. -1986- Sarcocapnos. Pp. 433-438. In: Castroviejo et al. (eds.). Flora Ibérica, vol. I. R. Jard. Bot, C.S.I.C. Madrid.

MAIRE R. -1965-Sarcocapnos. Pp. 10-19. In: Quézel. Flore de L'Afrique du Nord. Ed. Paul Lechevalier. Vol XII. Paris.

WILLKOMN, M. \& J. LANGE -1880- Prodromus Florae Hispanicae. Vol III. Stuttgart.

(Aceptado para su publicación en septiembre de 1991)

Dirección de la autora: Departamento de Biología Vegetal, Facultad de Biología. Universidad de Murcia, 30100 Murcia, España. 